\title{
Ferrocene Aryl Derivatives for the Redox Tagging of Graphene Nanoplatelets
}

\author{
Minjun Yang ${ }^{1}$, Christopher Batchelor-McAuley ${ }^{1}$, Luís Moreira Gonçalves ${ }^{2}$, Carlos F.R.A.C. Lima ${ }^{3,4}$, Vlastimil \\ Vyskočil $^{5}$, Kristina Tschulik ${ }^{1,6}$, Richard G. Compton ${ }^{1} *$ \\ ${ }^{1}$ Department of Chemistry, Physical and Theoretical Chemistry Laboratory, University of Oxford, South Parks Road, Oxford OX1 \\ 3QZ, United Kingdom \\ ${ }^{2}$ LAQV-REQUIMTE, Departamento de Química e Bioquímica, Faculdade de Ciências da Universidade do Porto, Rua do Campo \\ Alegre, s/n, 4169-007 Porto, Portugal \\ ${ }^{3}$ CIQ, Departamento de Química e Bioquímica, Faculdade de Ciências da Universidade do Porto, Rua do Campo Alegre, s/n, 4169- \\ 007 Porto, Portugal \\ ${ }^{4}$ Department of Chemistry \& QOPNA, University of Aveiro, Aveiro, Portugal \\ ${ }^{5}$ Charles University in Prague, Faculty of Science, University Research Centre UNCE "Supramolecular Chemistry", Department of \\ Analytical Chemistry, UNESCO Laboratory of Environmental Electrochemistry, Hlavova 2030/8, 12843 Prague 2, Czech Republic \\ ${ }^{6}$ Department of Analytical Chemistry - Micro-/Nano-Electrochemistry and Center for Electrochemical Sciences, Faculty of \\ Chemistry and Biochemistry, Ruhr-University Bochum, D-44780 Bochum, Germany
}

\begin{abstract}
Aryl derivatives of ferrocene are used for the modification of graphitic surfaces. Stronger adsorption is utilized as a route to enable the electrochemically tagging of new carbon materials. Model experiments are reported on an EPPG electrode where the adsorption of 1-(biphen-4-yl)ferrocene is found to be a factor of ca. 5 times more thermodynamically favorable than the underivatised form. Two further derivatives were also studied and the voltammetric responses of this class of ferrocenes are found to be significantly influenced by ion-pairing. Finally, the successful use of these new materials for the modification and redox tagging of graphene nanoplatelets is demonstrated.
\end{abstract}

\section{KEYWORDS}

Ferrocene, Physisorption, Voltammetry, Graphene Nanoplatelets, Ion-pairing

Address correspondence to Richard G. Compton, Richard.compton@chem.ox.ac.uk 


\section{Introduction}

Carbon materials have found increasing use in a wide range of analytical applications and energy technologies. In recent years this has been especially true for graphene based materials, [1] where due to their enhanced thermal and electrical conductivity, large surface area and mechanical strength they have been employed in a variety of uses. These applications include, but are not limited to: DNA or bio-labeling,[2,3] electroanalysis,[4,5] supercapacitors,[6] dye-sensitised solar cells[7] and nanomedicine.[8] However, isolated single layer graphene suffers from poor stability[9] and exhibits rippling (1 nm in height).[10] Graphene nanoplatelets, comprising of micron sized sheets of 2-10 nm (ref.[11]) in thickness may provide a plausible - more industrially suitable - alternative, and have importantly, already been demonstrated to be of distinct analytical use.[12]

The modification of carbon is commonly undertaken so as to instill the material with new or useful functionality. Historically, carbon surface modification with redox active species has been the focus of a significant amount of work. The most common methodologies employ the use of reactive species, such as diazonium salts[13,14] or carbon radicals formed from cleaved carbon/halide bonds. $[15,16]$ However, the application of such methods is inherently complicated by multi-layer formation as a result of polymerization. This polymerization leads to high surface coverages but the resulting voltammetry is commonly distorted.[17] Moreover, for graphene based materials the use of chemical bonding to the basal plane sites of the carbon may lead to a decrease in conductivity of the material due to breaking the material's aromatic structure. The use of physisorption for the modification of surface presents an attractive alternate methodology. Early work by Katz utilized pyrene and other aromatic tags to facilitate the adsorption of redox active species to carbon surfaces.[18,19] This methodology has been developed for application to the immobilization of a host of different biological systems, including proteins/enzymes,[19,20] DNA[21] and antibodies[22]. The technique has even be developed as a route by which graphene itself may be surface anchored to a substrate electrode.[23] Not only are such modification methods generally comparatively facile but the resulting surface coverages are inherently limited to being at maximum monolayer. Thus, much less distorted voltametric responses can be obtained.

Previous work has used long chain linkages between a ferrocene and a pyrene tag to facilitate adsorption to an highly ordered pyrolytic graphite (HOPG) surface,[24] or has alternatively focused on the direct adsorption of bi-functional redox species.[25] In contrast the present work utilizes a series aryl derivatised ferrocenes[26] without a linking chain for the modification of graphitic surfaces and graphene nanoplatelets. The presence of the aryl group is shown to enhance the ferrocene adsorption to the surface as monitored by the magnitude of the voltammetric response. Moreover, the redox potential for the differing ferrocene derivatives is shown to vary significantly by ca. hundreds of $\mathrm{mVs}$. This shift in potential is evidenced as relating to the differing ion pairing abilities of the electrochemically formed ferrocenium ions. 


\section{Experimental}

\section{Chemicals}

All chemicals (except the ferrocene derivatives) were provided by Sigma-Aldrich, St. Louis, MO, USA, at reagent grade unless specified otherwise. The graphene nanoplatelets (GNPs, $15 \mu \mathrm{m}$ wide, 6-8 $\mathrm{nm}$ thick) were purchased from Strem Chemicals, Newburyport, MA, USA. The Bamboo multi-walled carbon nanotubes were purchased from Nanolab, Waltham, MA, USA (BMWCNTs, 30 $\pm 10 \mathrm{~nm}$ wide, 5-20 $\mu \mathrm{m}$ long, and quoted specific surface area 200-400 $\mathrm{m}^{2} / \mathrm{g}$ ). All reagents were used without further purification.

From the SEM image analysis,[11] the average width of the used GNPs is $16.5 \pm 5 \mu \mathrm{m}$ ( sample population $=120$, bin size $=2 \mu \mathrm{m})$, whereas the average GNP area is $297 \pm 152 \mu \mathrm{m}^{2}$ (sample population $=62$, bin size $=50 \mu \mathrm{m}^{2}$ ). The thickness of graphene nanoplatelets is $7.1 \pm 2.0 \mathrm{~nm}$, in concordance with Strem's specification sheet. Using the experimentally derived thickness of $0.37 \mathrm{~nm} /$ graphene layer measured by Koh et al.[27] (cf. $0.34 \mathrm{~nm} / \mathrm{layer}$ in graphite), each GNP consists of ca. 16-22 graphene layers.

The ferrocene aryl derivatives were synthesized by the Suzuki-Miyaura cross-coupling reaction and characterized by ${ }^{1} \mathrm{H}$ and ${ }^{13} \mathrm{C}$ NMR spectroscopy, as previously described in the literature. ${ }^{21}$ The compounds were obtained in moderate to good yields (50-60\%) and were purified by recrystallization and sublimation under reduced pressure. The purity of the compounds was attested by gas chromatography showing a $\%(\mathrm{~m} / \mathrm{m})$ purity higher than $99 \%$ in all cases.

\section{General synthesis of 1-arylferrocenes}

A solution of $\mathrm{K}_{2} \mathrm{CO}_{3}(1.4 \mathrm{~mol} /$ equiv) in $12 \mathrm{~mL}$ of water per $1 \mathrm{mmol}$ of the limiting reactant was added to a solution of 1-bromoferrocene ( $1 \mathrm{~mol} /$ equiv), arylboronic acid (1.2 mol/equiv) and palladium (II) acetate, $\mathrm{Pd}(\mathrm{OAc})_{2},(2 \mathrm{~mol} \%)$ in the same volume of DMF. The resultant mixture was heated at $80{ }^{\circ} \mathrm{C}$ for $5 \mathrm{~h}$ under stirring. The final solution was allowed to cool to room temperature and extracted with dichloromethane. The organic layer was washed with water and aqueous $0.1 \mathrm{M} \mathrm{KOH}$, dried over anhydrous sodium sulfate and evaporated, yielding the product as impure 1-arylferrocene.

All aqueous solutions were prepared with deionized water of resistivity not less than 18.2 $\mathrm{M} \Omega \mathrm{cm}$ at $298 \mathrm{~K}$ (Millipore, Billerica, MA, USA). 0.10 M potassium chloride was used as the supporting electrolyte unless specified otherwise. 


\section{Equipment}

Voltammetric measurements were recorded using a $\mu$ Autolab II (Autolab, Utrecht, The Netherlands) with a standard three-electrode configuration. A saturated calomel electrode (SCE, ALS distributed by BASi, Tokyo, Japan) was used as the reference electrode and a graphite carbon rod as the counter electrode. Edge-plane pyrolytic graphite $(\mathrm{EPPG})($ radius $=2.10 \mathrm{~mm}$ ) and basal-plane pyrolytic graphite $($ BPPG) $($ radius $=2.75 \mathrm{~mm})$ were used as working electrodes.[28] These graphitic electrodes were made in house and were manufactured from highly ordered pyrolytic graphite (HOPG) (Le Carbone, Sussex, UK) of SP13 (ZYH) grade. By orientating the disc face perpendicular to the graphite crystal surface, an edge plane was achieved; alternatively a BPPG electrode could be formed by aligning the face parallel to the crystal surface. These graphite stubs were then set in custom made PTFE housing with an electrical connection made using a stainless steel core. All electrochemical experiments were performed in a three-necked flask placed inside a Faraday cage thermostated to $25{ }^{\circ} \mathrm{C}$. The EPPG was cleaned after each experiment using alumina slurries of decreasing particle sizes: 1.0-0.05 $\mu \mathrm{m}$ (Buehler, Lake Bluff, IL, USA), whereas the surface of the BPPG was peeled eight times using adhesive tape to maintain a flat surface.

\section{Modification}

Each electrode was immersed into a solution of ferrocene-derivative in acetonitrile for $30 \mathrm{~s}$ unless stated otherwise, then immersed into deionised water to wash away excess ferrocene to prevent the formation of microcrystals. Electrodes were dried by pure nitrogen gas before measurements.

$14.5 \mathrm{mg}$ of GNPs were suspended in $25 \mathrm{~mL}$ acetonitrile and $0.494 \mathrm{~mL}$ was taken out to mix with $1 \mathrm{~mL}$ of $2 \mathrm{mM}$ of ferrocene in acetonitrile then left to dry in desiccator for ferrocene absorption. The modified GNP was then transferred onto filter paper and abraded onto electrode by eight figure of eight cycles.[29] GNP suspension was shaken on a vortex (Whirlmixer, Loughbough, UK) before transfer into other vessels.

$6.6 \mathrm{mg}$ of BMWCNTs were suspended in $10 \mathrm{~mL}$ acetonitrile and $1 \mathrm{~mL}$ was removed to mix with $1 \mathrm{~mL}$ of $2 \mathrm{mM}$ of ferrocene in acetonitrile and then left to dry in a desiccator to realise adsorption. The modified BMWCNTs were then transferred onto filter paper and abrade onto electrode by eight figure of eight cycles. The BMWCNT suspension was shaken on a vortex prior to being transferred. 


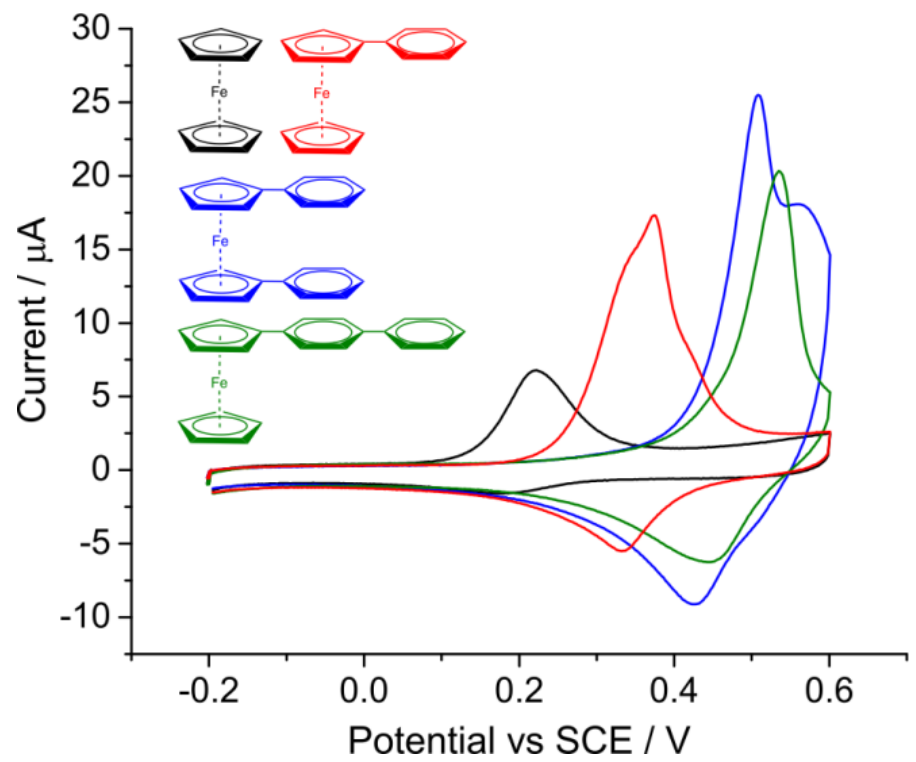

Figure 1: Voltammograms measured at an EPPG electrode modified with ferrocene absorbed directly on to the electrode surface via immersion of the electrode into a $5 \mathrm{mM}$ stock solution in acetonitrile for $30 \mathrm{~s}$. Also shown are the schematic structures of the various ferrocene derivatives (Black: ferrocene. Red: 1-phenylferrocene. Blue: 1,1'-diphenylferrocene. Green: 1 -(biphen-4-yl)ferrocene. Scan rate $=50 \mathrm{mV} \mathrm{s}^{-1}$. Electrolyte $=0.1 \mathrm{M} \mathrm{KCl}$

An EPPG electrode was immersed into a $5 \mathrm{mM}$ ferrocene solution (acetonitrile) for 30 seconds, after removal the electrode was rinsed and transferred to an aqueous electrochemical cell containing $0.1 \mathrm{M}$ $\mathrm{KCl}$. The electrode was subsequently voltammetrically scanned from $-0.2 \mathrm{~V}$ in an anodic direction to $+0.6 \mathrm{~V}$ and then swept back to $-0.2 \mathrm{~V}$. In the case of adsorption from a ferrocene $(\mathrm{Fc})$ containing solution a broad oxidative voltammetric wave was observed at $\sim+0.22 \mathrm{~V}$ (vs SCE). In the reverse scan a small reductive peak is observed. The oxidative feature is ascribed as being due to the one-electron oxidation of ferrocene to ferrocenium, as given by the equation:

$F C \rightleftharpoons \mathrm{FC}^{+}+e^{-}$

For the ferrocene derivatives qualitatively similar results are obtained in that for all cases a surface bound voltammetric wave is recorded exhibiting a diminished reductive peak. Most notably, however, the peak potentials for the different ferrocene-derivatives differ significantly with the oxidation of the 1-phenylferrocene occurring at $0.34 \mathrm{~V}, 1,1^{\prime}$-diphenyl occurs at $0.52 \mathrm{~V}$ and the 1-(biphen-4yl)ferrocene is oxidized at $0.54 \mathrm{~V}$. This is a large shift of over $0.32 \mathrm{~V}$ positive from that recorded for the underivatised ferrocene. The magnitude of the back peak is also found to follow a similar trend in that the size of the reduction wave (relative to the forward peak) decreases in the order biphenylferrocene $>$ diphenyl-ferrocene> phenyl-ferrocene> ferrocene.

The HOMO of ferrocene is predominantly non-bonding (A1g d-orbital)[30], consequently modification of the rings is unlikely to induce significant changes in the redox potential. The 
magnitude of the back-peak implies that the oxidised product is in some form consumed (cf. an EC reaction, where an electrochemical is followed by a chemical reaction step). It is noted that ferrocenium dimethanol is known to be stable on the voltammetric time scale in aqueous solution;[31] hence, it is concluded that the diminished back peak is not due to decomposition of the ferrocenium ion. Two alternative, non-mutually exclusive, explanations arise: Either ion-pairing[32,33] to the ferrocenium stabilizes it sufficiently that the reduction becomes thermodynamically unfavorable, hence the reduction would be gated; the formed ion pair must decouple prior to electron transfer in the reverse scan. Alternatively, the additional phenyl groups may decrease the solubility of the formed ferrocenium ion.[34] In both cases the potential shift of the peak would be expected to follow that found experimentally. In order to clarify the dominate mechanism by which the ferrocene voltammetric signal is shifted, the oxidation of the biphenyl-ferrocene was recorded in the presence of different electrolytes. SI Figure 1 depicts the variation of the voltammetric response in the presence of $\mathrm{KCl}, \mathrm{KNO}_{3}$ and $\mathrm{NaBF}_{4}$. In the presence of the poorly co-ordinating $\left[\mathrm{BF}_{4}\right]^{-}$anion the magnitude of the reductive peak is found to be significantly larger. From this it is concluded that the ion-pairing strength of the ferrocene derivatives has a dominant role upon the oxidation potential of the ferrocene group within the aqueous environment. Note that if the dissolution of the formed ferrocenium ion into solution had been the significant factor then a further diminished reductive peak would have been anticipated with the use of the poorly co-ordinating $\left[\mathrm{BF}_{4}\right]^{-}$anion since ion-pairing is expected to decrease the solubility of the ferrocenium ion.

Apart from the oxidation potential, a second notable difference between the voltammetric responses of the surface bound ferrocene-derivatives is the magnitude of the voltammetric peaks where in all cases for adsorption from a $5 \mathrm{mM}$ stock solution the aryl modified species exhibit larger voltamemtric responses. Importantly the difference between the magnitude of the adsorbate signal for the unmodified and derivatised ferrocenes is greater when measured on a BPPG electrode instead an EPPG (SI section 2).

Through the use of Faraday's first law and integration of the voltammetric peak the surface coverage of the adsorbed ferrocene can be directly determined. The surface coverage of both ferrocene and biphenyl-ferrocene were studied as a function of the concentration $(0.1-10 \mathrm{mM})$ of in the adsorption solution (acetonitrile). Figure 2 depicts the voltammetric response, where for both ferrocene and biphenyl-ferrocene the voltammetric peak current is found to scale with concentration. The ferrocene adsorption is taken to follow the Langmuir isotherm, such that, the surface coverage $(\Gamma)$ may be described using the following equation: [35]

$\Gamma=\frac{\Gamma_{\max } K_{e q}[F c]}{1+K_{e q}[F c]}$

where $\Gamma_{\max }$ is the maximum surface coverage, $\mathrm{K}_{\mathrm{eq}}$ is the adsorption equilibrium constant and [Fc] is the solution (acetonitrile) phase concentration of the ferrocene or ferrocene derivative. Figure $2 \mathrm{~b}$ ) shows the corresponding Langmuir plot of the inverse of the measured surface coverage against the 
inverse of concentration. Note it was confirmed that submersion of the electrode in the ferrocene containing acetonitrile solution for longer periods did not lead to higher surface coverages. From this Langmuir plot the maximum surface coverage of ferrocene and 1-(biphen-4-yl)ferrocene was

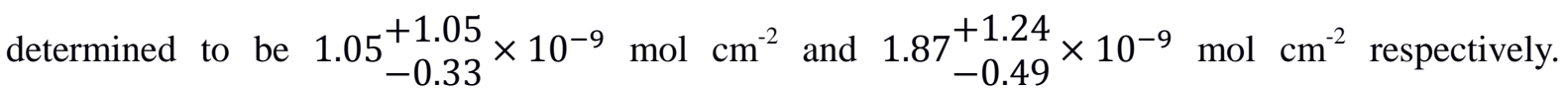
Consequently, within the accuracy of the experiment the maximum surface coverages of the two species are indistinguishable. Moreover, these values are, once the surface roughness of the graphitic surface is accounted for,[36] consistent with a near monolayer coverage of the adsorbed ferrocene.
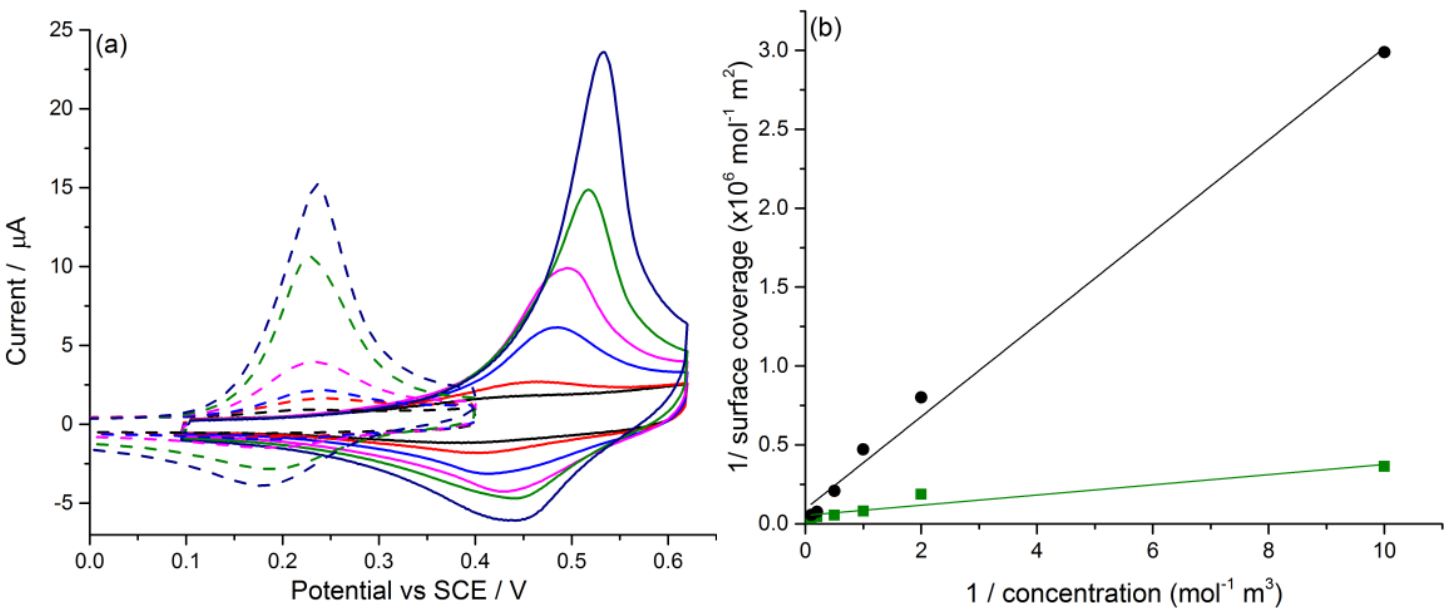

Figure 2: a) Voltammogram measured at an EPPG electrode with ferrocenes absorbed on to the electrode surface by immersion of the electrode into different concentration of ferrocene solution (0.1-10 mM; where the different colors represent different ferrocene (or derivative) concentrations) in acetonitrile for $30 \mathrm{~s}$ prior to being transferred into an aqueous electrochemical cell. Dashed: ferrocene. Solid line: 1-(biphen-4-yl)ferrocene. Scan rate = 50 $m V s^{-1}$. Electrolyte $\left.=0.1 \mathrm{M} \mathrm{KCl} . \mathrm{B}\right)$ Shows the Langmuir plot of 1/surface coverage against 1/concentration of ferrocene (black) or 1-(biphen-4-yl)ferrocene (green). Intercept and slope of the line of best fit provide a measure of the maximum surface coverage $\left(\Gamma_{\max }\right)$ and the adsorption constant $\left(K_{\text {eq }}\right)$.

Although the maximum surface coverages are comparable for the two species the adsorption thermodynamics are found to differ where the $K_{\text {eq }}$ values are $3.24+1.74 \times 10^{5} \mathrm{~mol}^{-1} \mathrm{~cm}^{3}$ and ${ }_{-0.72}^{+0.95} \times 10^{6} \mathrm{~mol}^{-1} \mathrm{~cm}^{3}$ for ferrocene and the 1-(biphen-4-yl)ferrocene species, respectively. Hence the adsorption of the 1-(biphen-4-yl)ferrocene to the graphitic EPPG surface is approximately five times more favorable than the underivatised ferrocene.

Having evidenced the enhanced ability of the ferrocene derivatives to adsorb upon macroscopic graphite surfaces, the work now turns to study the modification of graphene nanoplatelets (GNPs). Modification of the GNPs was achieved through suspension of the $0.28 \mathrm{mg}$ of GNPs in $1 \mathrm{~mL}$ of a 2 $\mathrm{mM}$ ferrocene (or ferrocene derivative) solution. The solvent was removed from the resulting suspension by evaporation and the resulting dry GNPs powder was subsequently abrasively attached 
to the EPPG surface.

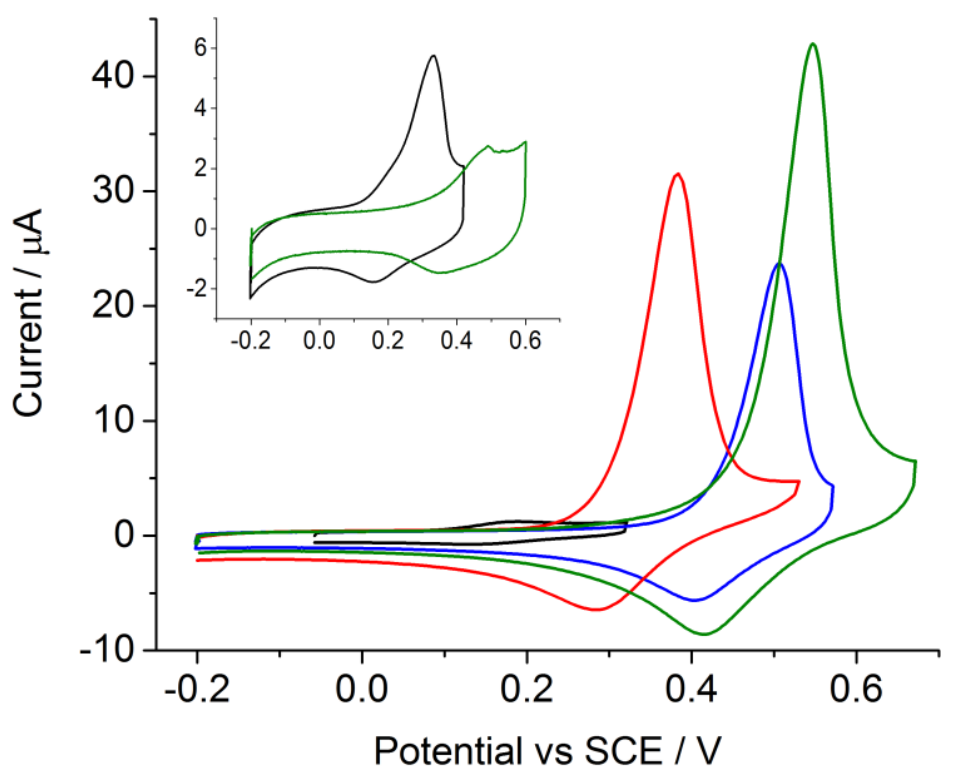

Figure 3: Voltammograms of ferrocene modified GNPs abrasively coated measured at an EPPG electrode using filter paper. Black: ferrocene. Red: 1-phenylferrocene. Blue: 1,1'diphenylferrocene. Green: 1-(biphen-4-yl)ferrocene. Inlay depicts the voltammetric response of abrasively attached BMWCNTs modified with Black: ferrocene and Green: 1-(biphen-4yl)ferrocene, note axis scales are the same as used for the main image. Scan rate $=50 \mathrm{mV} \mathrm{s}$. Electrolyte $=0.1 \mathrm{M} \mathrm{KCl}$

Figure 3 shows the voltammetric responses for the differing ferrocene modified GNPs. Using the abrasive methodology it is not possible to directly quantify the amount of GNP attached to the EPPG surface. However, repeat experiments showed the magnitude of voltammetric signal to fluctuate by $\mathrm{cf}$. $50 \%$. This variability in the magnitude of the peak current is ascribed as being related to the variability of the quantity of GNP attached to the surface during the abrasive modification procedure. Note that the other common electrode modification procedure - drop-casting - is not appropriate for the current investigation due to the use of volatile solvents for suspending the carbon materials, which will lead to the solvation and hence dissolution of the ferrocene species and loss from the GNP. For all three aryl derivatives the GNP material shows a large adsorption wave. In contrast only a small voltammetric feature is observed for the underivatised ferrocene GNPs (black line in Figure 3). The voltammetric signals for the ferrocene derivatives are 30-70 times larger than that found for the underivatised form. This significant difference in the magnitude of the peaks likely relates to the preferential adsorption of the ferrocene derivatives onto the basal sites present on the GNPs. Moreover, the inlay of Figure 3 shows the results for the adsorption of ferrocene and 1-(biphen-4yl)ferrocene, where although the magnitude of the underivatised ferrocene peak has increased roughly four-fold (peak current $\sim 1.2 \mu \mathrm{A}$ (GNPs) to $\sim 5.0 \mu \mathrm{A}$ (BMWCNTs)), the peak for the adsorbed 1(biphen-4-yl)-ferrocene has decreased to being virtually indistinguishable from the background 
capacitive current. The BMWCNTs exhibit a high surface coverage of edge plane sites, which is consistent with the observed decrease in the surface coverage of the adsorbed ferrocene as evidenced by the magnitude of the voltammetric signal.

\section{Conclusions}

The successful use of phenyl derivatised ferrocenes for the modification of carbon surfaces has been evidenced. The presence of the aryl groups serves to facilitate the surface adsorption of the ferrocene to the graphitic surface. On an EPPG electrode adsorption of the ferrocene compounds from an acetonitrile solution allows direct control of the coverage, the magnitude of the adsorption equilibrium constant is found to be five times larger for 1-(biphen-4-yl)ferrocenethan for ferrocene. For the modification of graphene nanoparticles distinctly larger $(>3,000 \%)$ surface bound voltammetric waves were observed for modification with the derivatised as opposed to the underivatised ferrocene. This methodology lends itself well for the redox tagging of new carbon nanomaterials, moreover the surface coverage is limited to being a monolayer or less, resulting in well-defined voltammetric signals.

\section{Acknowledgements}

The research leading to these results has received partial funding from the European Research Council under the European Union's Seventh Framework Programme (FP/2007-2013) / ERC Grant Agreement n. [320403].

C.F.R.A.C.L. and L.M.G. thank Fundação para a Ciência e a Tecnologia (FCT) for the award of the Research Grants SFRH/BPD/77972/2011 and SFRH/BPD/76544/2011, respectively. V.V. thanks the Grant Agency of the Czech Republic (Project GP13-23337P) for the financial support. K.T. acknowledges financial support by the State of NRW (NRW Rückkehrerprogramm) and the Cluster of Excellence RESOLV (EXC 1069) funded by the Deutsche Forschungsgemeinschaft. 


\section{References}

[1] Pumera, M. Electrochem. Comm. 2013, 36, 14-18.

[2] Bonanni, A.; Chua, C. K.; Zhao, G.; Sofer, Z. k.; Pumera, M. ACS nano 2012, 6, 8546-8551.

[3] HuilingáLoo, A. Nanoscale 2013, 5, 7844-7848.

[4] Seah, T. H.; Poh, H. L.; Chua, C. K.; Sofer, Z.; Pumera, M. Electroanalysis 2014, 26, 62-68.

[5] Chua, C. K.; Pumera, M. Electroanalysis 2013, 25, 945-950.

[6] Chen, J.; Li, C.; Shi, G. J. Phys. Chem. Lett. 2013, 4, 1244-1253.

[7] Miao, X.; Tongay, S.; Petterson, M. K.; Berke, K.; Rinzler, A. G.; Appleton, B. R.; Hebard, A. F. Nano Lett. 2012, 12, 2745-2750.

[8] Servant, A.; Bianco, A.; Prato, M.; Kostarelos, K. Bioorg. Med. Chem. Lett. 2014, 24, 1638-1649.

[9] Radich, J. G.; Krenselewski, A. L.; Zhu, J.; Kamat, P. V. Chem. Mat. 2014, 26, 4662-4668.

[10] Meyer, J. C.; Geim, A. K.; Katsnelson, M. I.; Novoselov, K. S.; Booth, T. J.; Roth, S. Nature 2007, 446, 6063.

[11] Poon, J.; Batchelor-McAuley, C.; Tschulik, K.; Compton, R. G. Chem. Sci. 2015, 6, 2869-2876.

[12] Lim, C. S.; Chua, C. K.; Pumera, M. Analyst 2014, 139, 1072-1080.

[13] Allongue, P.; Delamar, M.; Desbat, B.; Fagebaume, O.; Hitmi, R.; Pinson, J.; Savéant, J.-M. J. Am. Chem. Soc. 1997, 119, 201-207.

[14] Gam Derouich, S.; Rinfray, C.; Izzet, G.; Pinson, J.; Gallet, J.-J.; Kanoufi, F. d. r.; Proust, A.; Combellas, C. Langmuir 2014, 30, 2287-2296.

[15] Poizot, P.; Simonet, J. Electrochem. Comm. 2012, 23, 137-140.

[16] Jouikov, V.; Simonet, J. Electrochem. Comm. 2014, 46, 132-136.

[17] Li, Q.; Batchelor-McAuley, C.; Lawrence, N. S.; Hartshorne, R. S.; Compton, R. G. New J. Chem. 2011, $35,2462-2470$.

[18] Katz, E. J. Electroanal. Chem. 1993, 361, 109-114.

[19] Katz, E. J. Electroanal. Chem. 1994, 365, 157-164.

[20] Udit, A. K.; Hill, M. G.; Bittner, V. G.; Arnold, F. H.; Gray, H. B. J. Am. Chem. Soc. 2004, 126, 1021810219.

[21] Gorodetsky, A. A.; Barton, J. K. Langmuir 2006, 22, 7917-7922.

[22] Mahe, L. S. A.; Green, S. J.; Winlove, C. P.; Jenkins, A. T. A. J. Solid State Electrochem. 2014, 18, 32453249.

[23] Zong, X.; Kong, N.; Liu, J.; Yang, W.; Cao, M.; Gooding, J. J. Electroanalysis 2014, 26, 84-92.

[24] Anne, A.; Bahri, M. A.; Chovin, A.; Demaille, C.; Taofifenua, C. Phys. Chem. Chem. Phys. 2014, 16, $4642-$ 4652.

[25] Poon, J.; Batchelor-McAuley, C.; Tschulik, K.; Palgrave, R. G.; Compton, R. G. Nanoscale 2015, 7, 20692075.

[26] Lima, C. F. R. A. C.; Fernandes, A. M.; Melo, A.; Goncalves, L. M.; Silva, A.; Santos, L. B. Phys. Chem. Chem. Phys. 2015.

[27] Koh, Y. K.; Bae, M.-H.; Cahill, D. G.; Pop, E. ACS nano 2010, 5, 269-274.

[28] Banks, C. E.; Compton, R. G. Anal. Sci. 2005, 21, 1263-1268.

[29] Moore, R. R.; Banks, C. E.; Compton, R. G. Anal. Chem. 2004, 76, 2677-2682.

[30] Lein, M.; Frunzke, J.; Timoshkin, A.; Frenking, G. Chem. Eur. J. 2001, 7, 4155-4163.

[31] Batchelor-McAuley, C.; Gonçalves, L. M.; Xiong, L.; Barros, A. A.; Compton, R. G. Chem. Commun. 2010, 46, 9037-9039.

[32] Laborda, E.; Olmos, J. M.; Torralba, E.; Molina, A. Anal. Chem. 2015, 87, 1676-1684.

[33] Evans, D. H. Chem. Rev. 2008, 108, 2113-2144.

[34] Chevallier, F. G.; Klymenko, O. V.; Jiang, L.; Jones, T. G. J.; Compton, R. G. J. Electroanal. Chem. 2005, $574,217-237$

[35] Foo, K. Y.; Hameed, B. H. Chem. Eng. J. 2010, 156, 2-10.

[36] Neumann, C. C. M.; Batchelor-McAuley, C.; Downing, C.; Compton, R. G. Chem. Eur. J. 2011, 17, 73207326. 